\title{
Assessment of the voluntary activation of the diaphragm using cervical and cortical magnetic stimulation
}

\author{
T. Similowski, A. Duguet, C. Straus, V. Attali, D. Boisteanu, J-Ph. Derenne
}

\begin{abstract}
Assessment of the voluntary activation of the diaphragm using cervical and cortical magnetic stimulation. T. Similowski, A. Duguet, C. Straus, V. Attali, D. Boisteanu, J-Ph. Derenne. (C)ES Journals Ltd 1996.

ABSTRACT: The twitch occlusion technique is a promising tool for use in assessing central drive to the diaphragm and determining maximal transdiaphragmatic pressure $(P$ di) from submaximal efforts. Its clinical use is limited by difficulties inherent to bilateral electrical stimulation (BES) of the phrenic nerves. This study was designed to revisit the technique using cervical magnetic stimulation (CMS). In addition, the effects of a voluntary contraction on diaphragm response to magnetic stimulation of the cortex (CxMS) were studied.

Seven volunteers aged 23-33 yrs were studied. $P_{\text {di }}$ was determined at relaxed functional residual capacity $($ FRC) in response to BES $(P$ di,p-ES) and CMS $(P$ di,pCMS), and the effects of an increasing voluntary contraction $(P$ di,vol) were assessed. The same procedure was applied to CxMS.

$P$ di,p-CMS at relaxed FRC was $27.5 \pm 2.2 \mathrm{cmH}_{2} \mathrm{O}$ (mean \pm SEM), about $20 \%$ higher

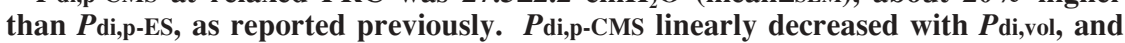
six out of seven subjects were capable of producing voluntary contractions sufficient to extinguish the twitch. More complex patterns were observed with CxMS.

Cervical magnetic stimulation provides diaphragmatic twitch occlusion data very similar to bilateral electrical phrenic stimulation. Magnetic stimulation, be it cervical or cortical, could probably be helpful for the assessment of central and peripheral mechanisms of diaphragmatic dysfunction in the clinical setting. Eur Respir J., 1996, 9, 1224-1231.
\end{abstract}

Laboratoire de Physiopathologie Respiratoire, Service de Pneumologie et Réanimation, Groupe Hospitalier Pitié-Salpêtrière, Paris, France.

Correspondence: T. Similowski

Laboratoire de Physiopathologie Respiratoire Service de Pneumologie et de Réamination Groupe Hospitalier Pitié-Salpêtrière 47-83, Bd de l'Hôpital 75651 Paris Cedex 13

France

Keywords: Cervical magnetic stimulation cortical stimulation

diaphragm

phrenic stimulation

twitch occlusion

Received: June 101994

Accepted after revision January 91996
The degree to which a skeletal muscle can be activated during attempted maximal voluntary efforts can be assessed using the technique of twitch interpolation. First described by MERTON [1], it involves the superimposition of twitches produced by electrical stimulation on a voluntary contraction. The amplitude of the response to peripheral nerve stimulation decreases monotonically as the strength of the underlying contraction increases. It is commonly held that this contraction stems from maximal activation when it completely suppresses the response to nerve stimulation $[1,2]$. Co-operative subjects are able to fully activate most limb muscles [1-3], but with regards to the diaphragm this issue has been controversial. A commonly accepted idea is that the diaphragm does not produce its maximal force during inspiratory manoeuvres, but rather during expulsive tasks when there is co-contraction of abdominal muscles $[4,5]$. Although conflicting results have been published [6], several studies applying the twitch occlusion technique to the diaphragm suggest that normal subjects can voluntarily produce maximal diaphragmatic contraction using inspiratory as well as expulsive efforts [7, 8]. This has been demonstrated in a comprehensive and elegant study by GANDEVIA and co-workers [9], who, in addition, first raised the possibility of testing maximal voluntary drive to respiratory muscles by motor cortical stimulation [10].
Besides these physiological considerations, the twitch occlusion technique could be highly relevant clinically, since it could be used to evaluate the intensity of the central command to the diaphragm. Indeed, in animals, estimates of central drive derived from twitch occlusion closely mirror central drive as reflected by recordings of phrenic nerve activity [11]. In humans, the twitch interpolation technique is the only test among those used to assess respiratory muscle function that can separate peripheral from central diaphragmatic fatigue or weakness $[12,13]$. In this respect, the failure of asthmatic patients to achieve maximal diaphragmatic activation [14], and the link of this failure to depression of mental state [15] have led to the idea that reduced voluntary drive to breathe could be a source of unexplained ventilatory failure in asthma. Conversely, the ability of patients with severe chronic obstructive pulmonary disease (COPD) to activate their diaphragm fully [16] argues against central inhibition of respiration as a mechanism of $\mathrm{CO}_{2}$ retention or respiratory failure.

Another salient feature of the twitch occlusion technique is that, because the response to nerve stimulation is linearly related to the intensity of the underlying contraction, it permits accurate assessment of maximal muscle strength under conditions where the patient is unable or unwilling to co-operate [7]. 
Table 1. - Characteristics of the subjects, and transdiaphragmatic pressure data at FRC

\begin{tabular}{llccccrr}
\hline $\begin{array}{l}\text { Subject } \\
\text { No. }\end{array}$ & $\begin{array}{c}\text { Age } \\
\text { yrs }\end{array}$ & Sex & $\begin{array}{c}\text { Height } \\
\mathrm{cm}\end{array}$ & $\begin{array}{c}\text { Weight } \\
\mathrm{kg}\end{array}$ & $\begin{array}{c}P_{\text {di,p-ES }} \\
\mathrm{cmH}_{2} \mathrm{O}\end{array}$ & $\begin{array}{r}P_{\text {di,p-CMS }} \\
\mathrm{cmH}_{2} \mathrm{O}\end{array}$ & $\begin{array}{r}P_{\text {di,max }} \\
\mathrm{cmH}_{2} \mathrm{O}\end{array}$ \\
\hline 1 & 29 & $\mathrm{M}$ & 165 & 65 & 25.2 & 30.4 & 113.8 \\
2 & 30 & $\mathrm{~F}$ & 175 & 62 & 20.5 & 23.0 & 76.8 \\
3 & 28 & $\mathrm{~F}$ & 166 & 64 & 22.6 & 27.2 & 123.8 \\
4 & 23 & $\mathrm{~F}$ & 163 & 52 & 17.6 & 20.1 & 79.0 \\
5 & 25 & $\mathrm{M}$ & 165 & 65 & 19.8 & 24.5 & 159.8 \\
6 & 33 & $\mathrm{M}$ & 187 & 85 & 31.5 & 35.0 & 175.0 \\
7 & 28 & $\mathrm{M}$ & 165 & 70 & 26.0 & 32.0 & 155.0 \\
\hline Mean & & & & & 23.3 & 27.5 & 126.2 \\
SEM & & & & & 1.9 & 2.2 & 16.0 \\
\hline
\end{tabular}

FRC: functional residual capacity; M: male; F: female; $P$ di,p-ES: amplitude of the transdiaphragmatic twitch pressure elicited by bilateral electrical phrenic nerve stimulation at relaxed FRC; $P_{\text {di,p-CMS: amplitude of the }}$ transdiaphragmatic twitch pressure elicited by cervical magnetic stimulation at relaxed FRC; $P$ di,max: amplitude of the transdiaphragmatic twitch pressure generated by maximal, static, pure diaphragmatic contractions.

For all these reasons, the twitch occlusion technique has been endorsed by the 1989 National Heart, Lung and Blood Institute (NHLBI) workshop on respiratory muscle fatigue as a potentially major diagnostic tool [17]. However, its usefulness is restricted by difficulties inherent to bilateral supramaximal stimulation of the phrenic nerves [17]. The present study was, therefore, designed to revisit the assessment of the voluntary drive to the diaphragm using noninvasive and easy to use cervical and cortical magnetic stimulations.

\section{Material and methods}

\section{Subjects}

Seven healthy volunteers ( 4 males and 3 females; aged 23-33 yrs) participated in the study (table 1), after approval of the Local Ethical Committee. Two of them had previous experience of respiratory muscle experiments, considerable in one case. All subjects were studied sitting on a chair, abdomen unbound. They had been informed of the purpose and methods of the study.

\section{Measurements}

Electromyograms. Surface recordings of the right and left costal diaphragmatic electromyographic activity (Rdi and Ldi, respectively) were obtained using skin-taped silver cup electrodes placed in the 7th and 8th right and left intercostal spaces and connected to a Dantec 2000 electromyograph (Dantec Electronik, Denmark). In four subjects, a surface electromyogram of one sternomastoid muscle was also obtained (Esm) (see Discussion).

Pressures. Oesophageal and gastric pressure $(P$ oes and $P$ ga) were measured with two balloon-catheters (thinwalled balloons sealed over a distally side-holed polyethylene catheter, $50 \mathrm{~cm}$ length and $1.7 \mathrm{~mm}$ internal diameter $(I D))$. Transdiaphragmatic pressure $(P$ di $)$ was obtained on-line by connecting these catheters to a Validyne MP45 differential pressure transducer $\left( \pm 200 \mathrm{cmH}_{2} \mathrm{O}\right.$; Validyne,
Northridge, USA). Pressure signals were fed to a personal computer and digitized through a Data Translation ${ }^{\circledR}$ DT 2801-A A/D board. Pdi was continuously displayed to the subject on a Hewlett-Packard oscillograph.

Rib cage $(R C)$ and abdomen $(A B)$ displacements. $\mathrm{RC}$ and $\mathrm{AB}$ displacements were studied in two subjects (Nos 6 and 7). Magnetometry and inductance plethysmography being unreliable during magnetic stimulation, tightly fitting air-filled tubings were taped around the upper rib cage and abdomen and connected to two differential pressure transducers ( $\pm 2 \mathrm{cmH}_{2} \mathrm{O}$; Validyne, Northridge, USA). Their position was adjusted during diaphragmatic twitches produced by selective bilateral electrical stimulation of the phrenic nerves (see below). The AB tubing was set approximately at the level of the umbilicus, in such a way as to record a positive change in pressure during diaphragmatic twitches (i.e. AB expansion). The $\mathrm{RC}$ tubing was set as high as possible on the rib cage, in such a way as to record a negative change in pressure during diaphragmatic twitches (i.e. upper rib cage inward movement).

\section{Stimulations}

Bilateral electrical stimulation (BES) of the phrenic nerves was performed according to the usual technique, using bipolar electrodes connected to a constant current stimulator delivering square-wave shocks of $0.1 \mathrm{~ms}$ duration (Curamètre®; Bio-Industry, Outreau, France). The nerve once spotted, the intensity of BES was progressively increased until a plateau in the amplitude of the corresponding compound diaphragmatic action potentials (CDAP) was reached. It was then further increased by $10-20 \%$, to ascertain supramaximality.

Magnetic stimulation was carried out using a first generation Magstim 200 stimulator (The Magstim Co., Sheffield, UK) equipped with a $90 \mathrm{~mm}$ circular coil (1.5 Tesla, $0.1 \mathrm{~ms}$ square-wave pulses). Cervical magnetic stimulation (CMS) was performed as reported previously [18]. As with BES, its supramaximal nature was judged according to the relationship between stimulation intensity and CDAP amplitude. Because a plateau in CDAP was not always observed, the peak-to-peak amplitudes 


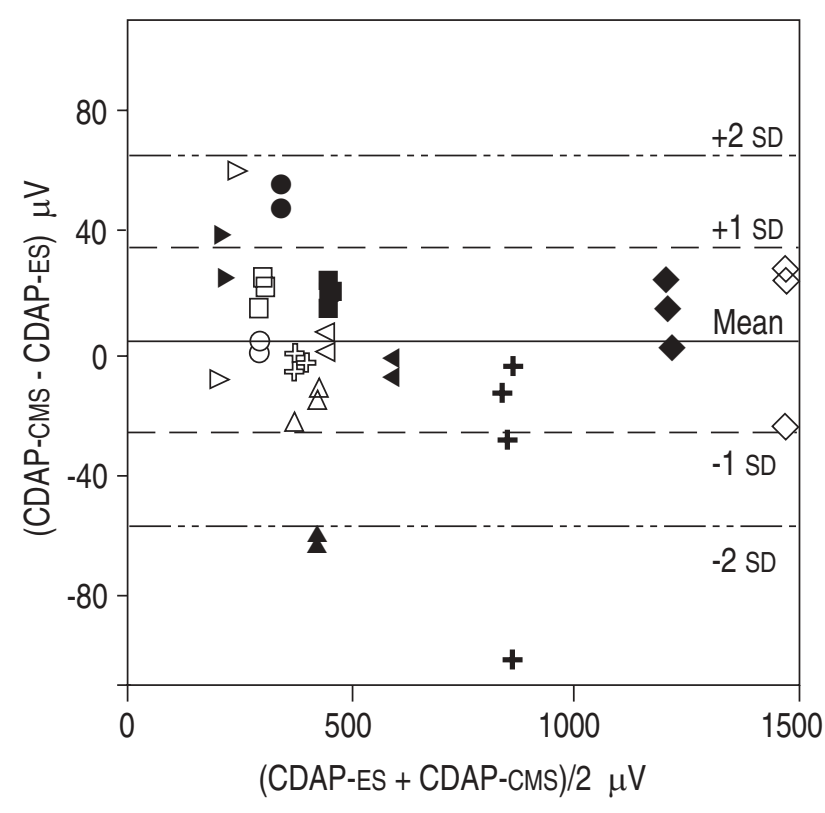

Fig. 1. - Bland and Altman [19] comparison of the peak-to-peak amplitude of compound diaphragmatic action potentials (CDAP) obtained with cervical magnetic stimulation (CMS) at maximal intensity (CDAP-CMS) with the peak-to-peak amplitude of CDAP obtained with supramaximal electrical stimulation (CDAP-ES). Each symbol corresponds to an individual subject ( 2 to 4 data points per subject). Open symbols correspond to the right hemidiaphragm, whereas closed symbols correspond to the left hemidiaphragm. The absence of significant difference between CDAP-CMS and CDAP-ES is an argument for the supramaximal nature of CMS. See text for details. $\square$ and $\mathbf{\square}$ : No. 1; $\bigcirc$ and $\bullet$ : No. $2 ; \Delta$ and $\boldsymbol{\Delta}$ : No. $3 ; \triangleright$ and $\triangleright:$ No. 4; ; and $\mathbf{+}$ : No. 5; $\triangleleft$ and $\triangleleft$ : No. 6; $\triangleleft$ and $\gg$ : No. 7

of the CMS-related CDAPs (maximal intensity) were systematically compared with those of the supramaximal BES-related CDAPs (fig. 1).

Magnetic stimulation of the cortex (CxMS) was performed with the coil positioned over the vertex, 1 to 3 $\mathrm{cm}$ behind the mid-auricular plane [20], at maximal stimulation intensity. The electromyographic (EMG) response to $\mathrm{CxMS}$ is hencerforth termed motor evoked potential (MEP).

The $P$ di responses to BES, CMS, and CxMS will henceforth be termed $P$ di,p-ES, $P$ di,p-CMS and $P$ di,c, respectively ("p" standing for peripheral, and "c" for central).

\section{Procedures}

Training. Before the experiments, all subjects were allowed time enough to learn how to generate and control $P$ di using visual feedback. Maximal $P$ di $\left(P_{\text {di,max }}\right)$ was then determined [5].

Stimulations. To preclude twitch potentiation, the subjects were first asked to breathe quietly and remain relaxed for 20 min [21]. BES, CMS and CxMS were performed at functional residual capacity (FRC), after airway occlusion, with the diaphragm relaxed. CMS and CxMS were then superimposed upon graded isovolumetric diaphragmatic contractions $(P \mathrm{di}, \mathrm{vol})$ ranging $10-100 \%$ of $P$ di,max.

Values in the results section are mean \pm standard error of the mean. a) $\mathrm{RC}$

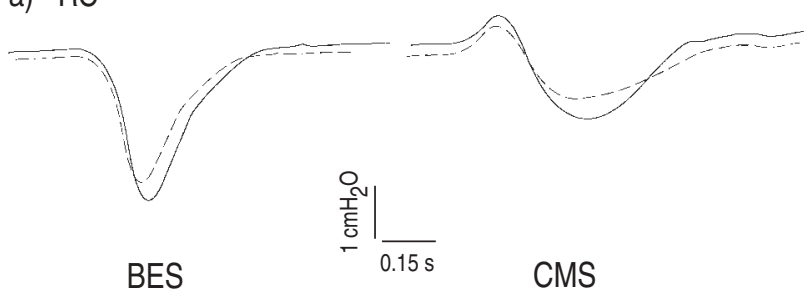

b) $\mathrm{AB}$
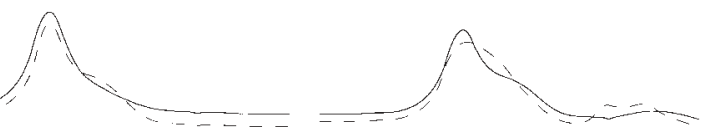

Fig. 2. - Representative examples of: a) rib cage (RC); and b) abdominal $(\mathrm{AB})$ displacements following bilateral electrical phrenic nerve stimulation (BES left tracings) and cervical magnetic stimulation (CMS right tracings) in two subjects (Nos. 6 and 7). — : subject No. 6; : subject No. 7.

\section{Results}

\section{Peripheral stimulation}

With increasing CMS intensity, a clear plateau was observed in all cases with respect to $P$ di,p-CMS, but only in four with respect to CDAPs. Figure 1, according to Bland and Altman [19], shows that the peak-to-peak amplitudes of the CDAPs produced by CMS at maximal intensity were not different from the peak-to-peak amplitudes of the CDAPs produced by supramaximal BES. Therefore, it is likely that supramaximality was consistently achieved with CMS in this series of experiments.

At FRC, with the diaphragm relaxed, mean \pm sem $P$ di,pCMS was $27.5 \pm 2.2 \mathrm{cmH}_{2} \mathrm{O}$, whereas $P$ di,p-ES was $23.3 \pm 1.9$ $\mathrm{cmH}_{2} \mathrm{O}$ (table 1). The difference was entirely accounted for by higher twitch $P$ oes values.

Phrenic conduction time ranged 5.5-7 $\mathrm{ms}$, and was similar with BES and CMS. In some subjects, the shape of the CMS-related CDAP was different from that of the BES-related CDAP.

In the two subjects in whom they were studied (Nos. 6 and 7), AB displacements induced by BES and CMS were similar in pattern and amplitude. BES-related RC displacements consisted in sharp paradoxical inward movements starting from the relaxed position. The RC pressure signal in response to CMS began with a slight increase, which was followed by a negative swing ascertaining an inward movement, but the latter was of lower amplitude and slower time course than with BES (fig. 2).

When CMS was superimposed upon a voluntary diaphragmatic contraction, the amplitudes of the corresponding twitches linearly decreased according to:

$$
P \text { di,p-CMS }=\text { a }- \text { b } P \text { di,vol }
$$

The Y-intercept "a" of Equation (1) amounted to 26.6 22.1 $\mathrm{cmH}_{2} \mathrm{O}$ (range 19.7-33.4), not significantly different from the actual $P$ di,p-CMS at FRC (table 1; paired t-test). The slope "b" of Equation (1) was $0.24 \pm 0.03$.

Individual $P$ di,p-CMS - $P$ di,vol relationships are depicted in figure $3 a$. In six subjects, $P$ di,p-CMS could be brought to zero, suggesting maximal diaphragmatic activation $[7,8]$. In subject No. 5 , a positive $P$ di,p-CMS persisted whatever the effort made (see Discussion). 
a)
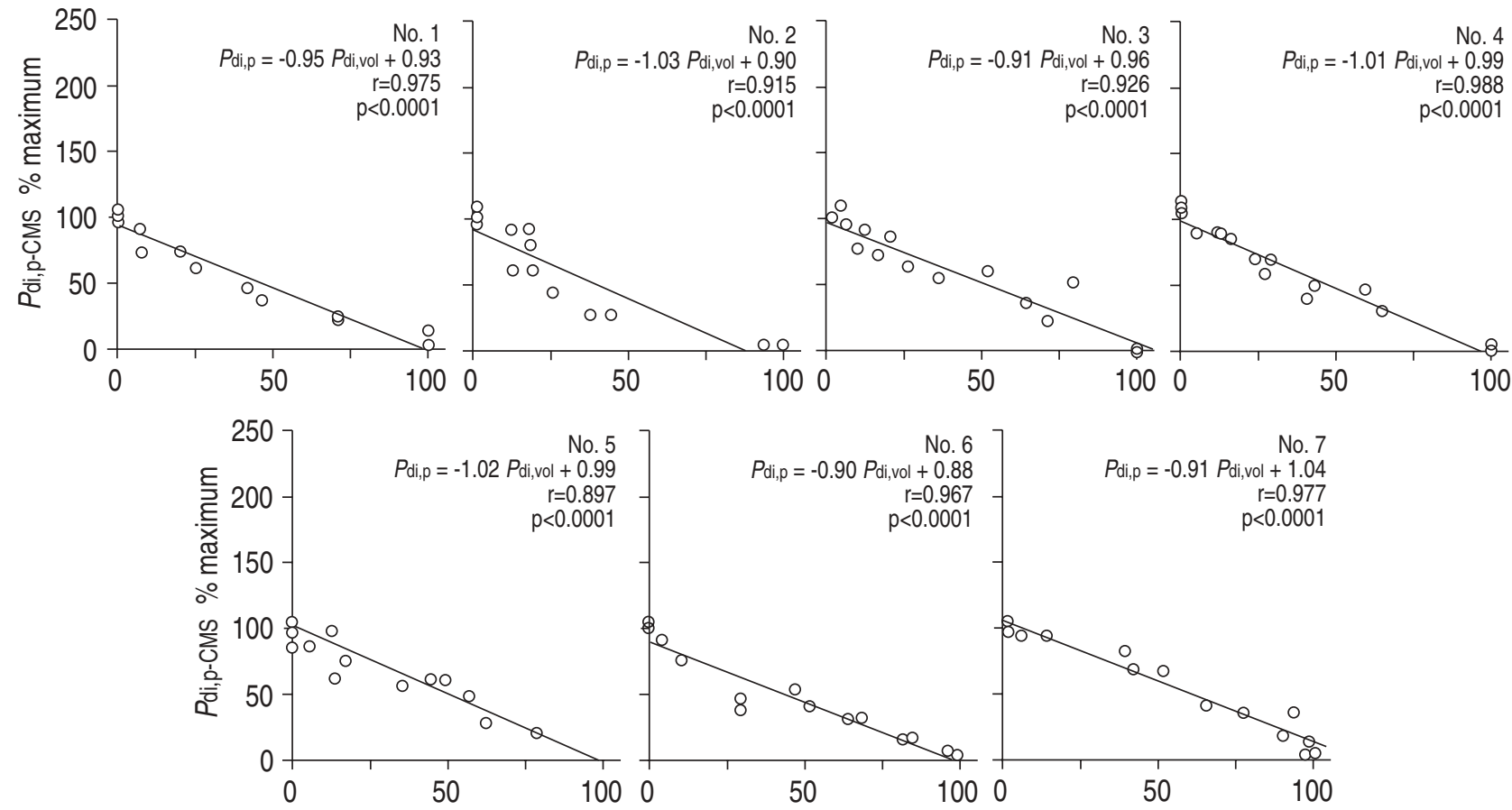

Pdi,vol \% maximum

b)
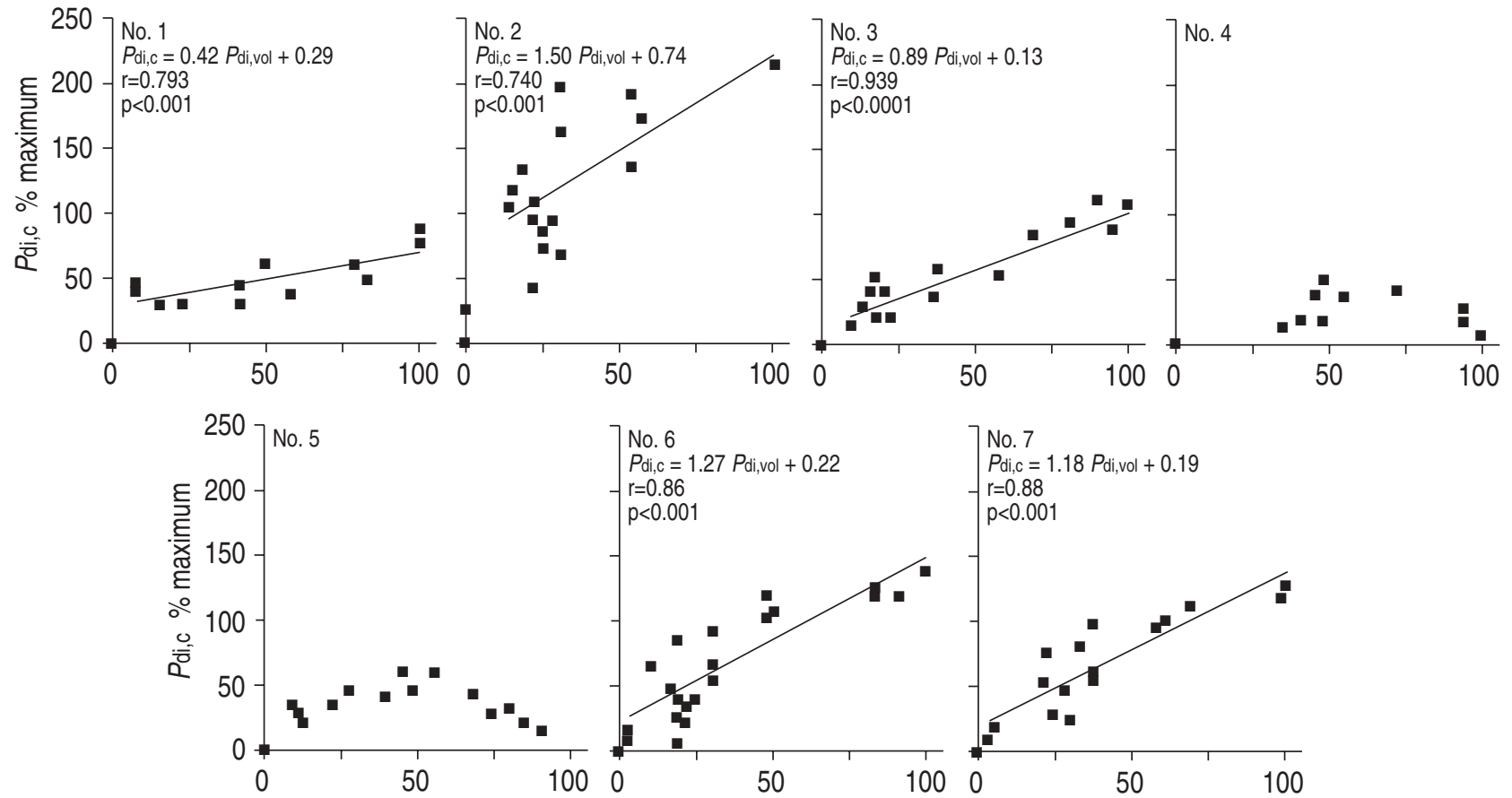

$P$ di,vol \% maximum

Fig. 3. - a) Effects of a voluntary diaphragmatic contraction $(P$ di,vol $)$ on the amplitude of the transdiaphragmatic pressure response to cervical magnetic stimulation $(P$ di,p-CMS $)$, in the seven subjects. The data points correspond to the expression both of $P$ di,vol and $P$ di,p-CMS as percentage of their maximum values. All subjects but No. 5 were able to produce a Pdi,vol extinguishing the response to peripheral phrenic nerve stimulation. b) Effects of a voluntary diaphragm contraction $(P \mathrm{di}, \mathrm{vol})$ on the amplitude of the transdiaphragmatic pressure response to cortical magnetic stimulation $\left(P_{\mathrm{di}, \mathrm{c}}\right)$, in the seven subjects. $P_{\mathrm{di}, \mathrm{vol}}$ is expressed as percentage of its maximal value. $P_{\mathrm{di}, \mathrm{c}}$ is expressed in percentage of $P_{\mathrm{di}, \mathrm{p}-\mathrm{CMS}}$ at relaxed FRC. In subjects Nos. 1-3, 6 and $7, P$ di,c continuously increased with $P_{\text {di,vol }}$ and could be fitted by a linear function. In subjects Nos. 4 and 5 , $P$ di,c first increased with $P$ di,vol (facilitation) and then decreased (twitch occlusion) (see text for details). 
a) CMS

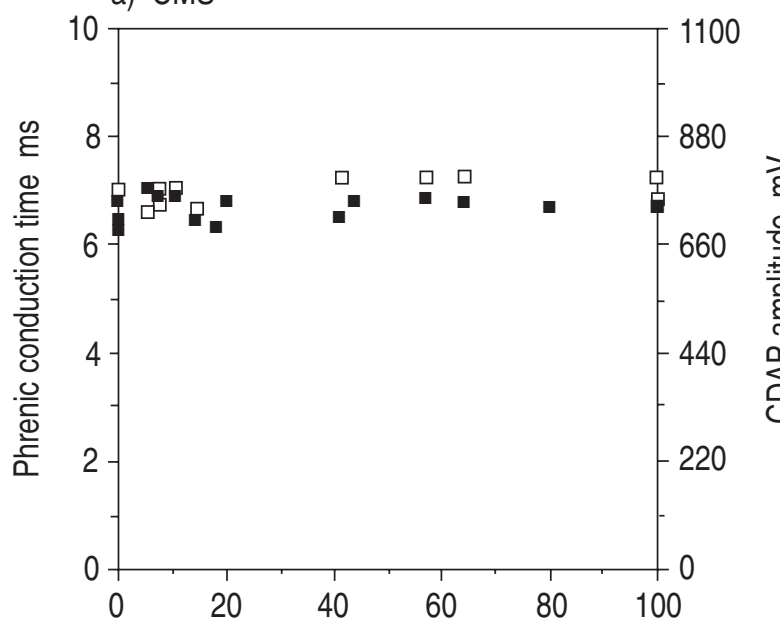

b) CxMS

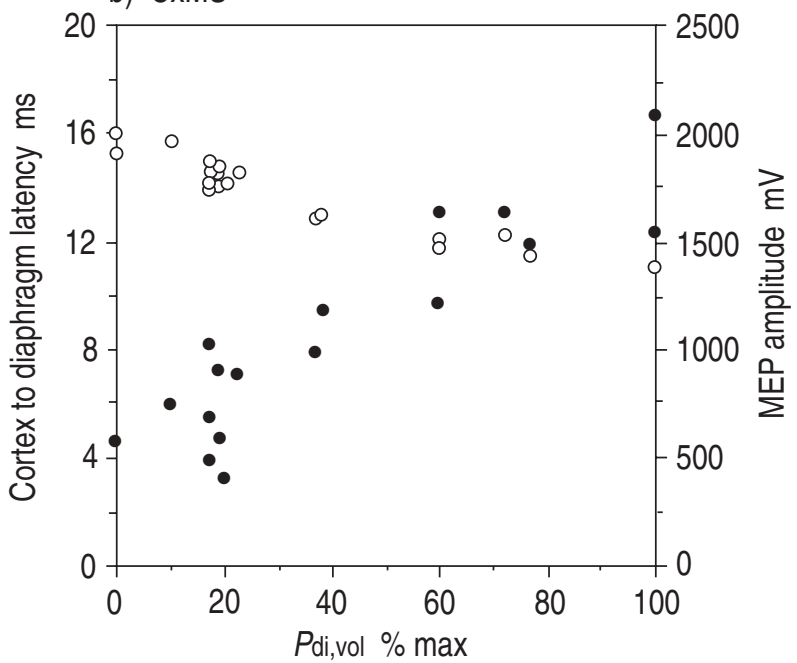

Fig. 4. - a) A representative pattern of the effects of a voluntary diaphragm contraction (Pdi,vol, expressed as percentage maximum) on the phrenic conduction times (open symbols) and on the amplitude of the compound diaphragmatic action potentials (CDAP) (closed symbols) recorded after cervical magnetic stimulation. b) The conduction times (open symbols) and the amplitude of the motor evoked potentials (MEP) (closed symbols) recorded after magnetic stimulation of the cortex (CxMS). With CxMS, the increase in amplitude of MEPs and the decrease in latency correspond to the phenomenon of facilitation.

$P$ di,vol did not affect the amplitude and the latency of the CDAPs (fig. 4).

\section{Cortical magnetic stimulation}

CxMS activated several upper and lower limb muscle groups. No response of the relaxed diaphragm was observed, and a $P$ di,vol of about $10 \% P$ di,max was needed for this response to appear.

In subjects Nos. 1-3, 6 and 7, $P$ di,c continuously increased with $P$ di,vol (fig. $3 b$ ), according to:

$$
P \text { di,c }=\mathrm{c}+\mathrm{d} P \text { di,vol }
$$

As a result, $P$ di,c twitches persisted at $P$ di,vol levels corresponding to the suppression of $P$ di,p-CMS. In subjects
Nos. 6 and 7, these twitches were associated with an outward $\mathrm{AB}$ displacement and a complex $\mathrm{RC}$ response, including an inward movement, making a diaphragmatic contribution likely (see Discussion).

It should be noted that because the absence of response of the relaxed diaphragm was probably due to the lack of power of the stimulator and not to a physiological mechanism [22], the corresponding data points have been discarded in order to compute the regression equations given in figure $3 \mathrm{~b}$. The slope of Equation (2) reached significance in the five subjects. Of note, the $r$ values were lower for CxMS than for CMS, but were not improved by fitting nonlinear models (e.g. power function) to the data.

With increasing $P$ di,vol, the amplitude of the MEPs increased and multiple EMG responses could be observed, whereas latencies tended to decrease (fig. 4).

In subjects Nos. 4 and 5, $P$ di,c initially increased with $P$ di,vol, and then markedly decreased (fig. $3 b$ ).

\section{Discussion}

The main finding of this study is that CMS can provide diaphragmatic twitch occlusion data very similar to those obtained with BES. The study further suggests that, given that some points are elucidated, CxMS could be used in the assessment of the central drive to respiratory muscles. In addition, it provides information which may contribute to explain the differences in $P$ di observed with CMS and BES. This aspect will be examined first.

Contribution to the understanding of the differences between CMS and BES

CMS is a noninvasive and easy-to-use nonvolitional test of diaphragmatic contraction [18, 23]. It is associated with $P$ di values higher than those obtained with BES, due to a larger $P_{\text {oes }}$ component ([23], and this study). Because CMS provokes diaphragmatic contraction via stimulation of cervical roots rather than of the phrenic nerve itself, it implies co-contraction of various muscles innervated by the $\mathrm{C}_{3}-\mathrm{C}_{6}$ roots [24], as well as muscles innervated by the XIth cranial nerve. Therefore, it has been postulated that the better "efficiency" of CMSinduced diaphragmatic contraction in terms of $P$ oes was due to decreased rib cage distortability as a result of the prior action of extradiaphragmatic muscles (e.g. sternomastoid or scaleni muscles) [23, 25].

There are other possibilities: 1) a direct contribution of CMS-related neck muscle contraction to $P$ oes (for such an explanation to hold, it has to be assumed that the $P$ oes produced by neck muscles contraction is not or is only partially transmitted to the abdominal cavity, and can therefore contribute to the build-up of $P \mathrm{di}$ ); or 2) partial recruitment of the diaphragm by BES due to incomplete aggregation of fibres constituting the phrenic nerve at the site of stimulation in the neck (this can be due to anatomical variability: on the one hand, the phrenic nerve in the neck is at times made of a complex of rootlets that unite to constitute a single nerve only in the thorax [26]; on the other hand an accessory phrenic nerve from the fifth cervical root sometimes adds to the main trunk 
in the mediastinum [27]. In both cases, CMS would activate all phrenic fibres, but not BES).

Our AB and RC displacement data in subjects Nos. 6 and 7 (fig. 2), showing a reduced and slower inward movement of RC with CMS, substantiate the rib cage stabilization hypothesis. In this respect, the initial positive pressure "bump" observed at the very beginning of $\mathrm{RC}$ movements as assessed by the devices used in this study is interesting to discuss. If it corresponded to RC expansion resulting from the action of neck muscles, there would be a direct contribution of the latter to $P_{\mathrm{oes}}$, and the CMS induced $P_{\text {oes }}$ swing would probably be biphasic [18]. Such a pattern is not observed in practice. Rather, a small positive $P$ oes deflection often precedes the CMS induced $P$ oes negative swing (see, for example, fig. 2 in [23]). Rib cage stabilization with CMS could, therefore, be the result of initial expiratory action on the rib cage, via contraction of the trapezius or pectoralis muscles for example. The increase in volume of these muscles associated with their contraction would then explain the initial positive RC "bump".

\section{Effects of an underlying contraction on the diaphragm response to $C M S$}

$P$ di,p-CMS linearly decreased with increasing $P$ di,vol, whereas the latency and the amplitude of the corresponding CDAPs did not change. Such a pattern confirms that CMS produces diaphragmatic responses through stimulation of peripheral nervous structures (the roots) rather than of a central one (the spinal cord). In the latter case, increasing $P$ di,p-CMS and increasing amplitude of CDAPs would have been expected with $P$ di,vol, together with reduced CDAP latency, as a result of spinal facilitation [28].

As stated in the introduction, the twitch occlusion technique has interesting potentialities for studies of diaphragmatic function [17]. However, the consistency of the stimulus delivered to the nerves by BES is often difficult to maintain during intense inspiratory manoeuvres recruiting neck muscles. Being by nature much easier to maintain than BES in this setting, CMS could promote the clinical use of information derived from the twitch occlusion. We feel that the data provided by this study are a step in this direction. Indeed, the influence of $P$ di,vol on $P$ di,p-CMS was comparable to what is described with BES: 1$)$ the y-intercept of the $P$ di,vol $-P$ di,p-CMS relationship $\left(26.6 \pm 2.1 \mathrm{cmH}_{2} \mathrm{O}\right)$ was not significantly different from the $P$ di,p-CMS values obtained at relaxed FRC $\left.\left(27.5 \pm 2.2 \mathrm{cmH}_{2} \mathrm{O}\right) ; 2\right)$ the slope of the relationship, corresponding to the twitch-to-tetanus ratio, was $0.24 \pm 0.03$, which is very close to values reported for the diaphragm and other mammalian muscles [29]; and 3) 6 out of 7 subjects were able to produce voluntary efforts suppressing the response to CMS. As we are confident that CMS was actually supramaximal (see Results), this suggests that these efforts were produced by maximal or close to maximal diaphragmatic command (see below). In addition, we performed a BES twitch occlusion procedure in subject No. 5, who failed to extinguish $P$ di,pCMS; the results were impossible to distinguish from those for CMS.

Given that CMS provides valid diaphragmatic twitch occlusion data, and taking into account the differences between $P$ di,p-ES and $P$ di,p-CMS discussed above, one could further submit that the "magnetic" twitch occlusion could in fact give a more realistic description of diaphragmatic properties than the "electric" one. The reasons for this contention are as follows. When $P$ di manoeuvres are performed with visual feedback, neck muscles often stay relaxed during low intensity contractions, and are coactivated during high intensity ones. BELLEMARE and BIGLAND-RITCHIE [7] reported that neck muscle contractions became significant for $P$ di,vol values of about $70 \%$ of $P$ di,max, and then increased with $P$ di,vol. In the four subjects where Esm was recorded (see Methods), we observed such a recruitment pattern. Neck muscle activation should markedly decrease upper rib cage distortability. Thus, during a BES twitch occlusion procedure, the stimulation-induced diaphragmatic contraction will interact with a highly distortable rib cage at low $P$ di,vol values, and with a much stiffer rib cage at high $P$ di,vol values.

The corresponding improved efficiency of diaphragmatic contraction probably explains the alinearity observed by Bellemare and Bigland-Ritchie [7] in the Pdi,p$P$ di,vol relationship. They noted that the slope of this relationship abruptly decreased after approximatively $70 \%$ of $P$ di,max (see fig. 7 in [7]), which led them to state that $P$ di,max extrapolated from submaximal efforts using the twitch occlusion principle could slightly underestimate the actual value. The CMS-related rib cage stabilization discussed previously should result in a more homogeneous "environment" for diaphragmatic contraction at all levels of $P$ di,vol and, therefore, reduce this problem. In most of our subjects (fig. 3a), we did not observe the slope change described by BELLEMARE and BIGLAND-RITCHIE [7], and the extrapolated values for $P$ di,max closely matched the actual values observed during attempted maximal efforts (126.2 \pm 16 vs $122.2 \pm 19.2 \mathrm{cmH}_{2} \mathrm{O}$, respectively; NS).

Effects of an underlying contraction on the diaphragmatic response to cortical magnetic stimulation

To our knowledge, all published studies dealing with diaphragmatic response to CxMS [20, 30-32] mention the need of a voluntary contraction for a diaphragmatic response to appear. This is probably simply a consequence of the insufficient power of first generation stimulators used in previous studies, as well as in the present one.

The increase in $P$ di,c with $P$ di,vol depicted in figure $3 b$, together with the shortening in latency and the increase in amplitude of MEPs depicted in figure 4, is consistent with the phenomenon of facilitation which has been well-described for other muscles [28]. However, $P_{\text {di,c }}$ continued to increase with $P$ di,vol over the whole range of $P$ di,vol studied in 5 out of 7 subjects (fig. $3 b$ ). This represents an important difference from limb muscles, where it is generally admitted that a voluntary contraction amounting to $10-20 \%$ of maximal strength is sufficient to maximize the effects of facilitation [33]. Only two subjects (Nos. 4 and 5) exhibited a somewhat expected pattern, namely increasing $P$ di,c up to a certain level of $P$ di,vol (facilitation) followed by a tendency for $P$ di,c 
to decrease (twitch occlusion). The disappearance of a cortical twitch with maximal voluntary activation of the corresponding muscle can indeed be inferred from the data published by MERTON et al. [10]. Two kinds of explanation could be proposed to account for the difference between the behaviour of the diaphragm and of other muscles.

Firstly, mechanisms of diaphragmatic facilitation could differ from those of limb muscles facilitation. In fact, facilitation in limb muscles depends partly on the preactivation of spinal motoneurones by information carried by Ia fibres [34]. Since the diaphragm contains few spindles, it is conceivable that motoneurone preactivation requires higher levels of tension than for limb muscles. Such a hypothesis seems to be supported by the continuous decrease of MEP latency with $P$ di,vol, suggesting persistent increasing facilitation.

Secondly, the role of muscles other than the diaphragm could be invoked. In this respect, the progressive recruitment of inspiratory and expiratory muscles during $P$ di,vol manoeuvres of increasing intensity would lead to their increasingly stronger contraction in response to CxMS, again because of the occurrence of facilitation. This contraction, under certain conditions, could contribute to $P$ di,c. Gandevia et al. [9] have already reported the persistence of $P$ di,c at $P$ di,vol levels where the peripheral response to phrenic nerve stimulation was abolished. Using BES, they have shown that normal, motivated subjects can produce diaphragmatic contractions intense enough to extinguish the twitch, both during expulsive and inspiratory efforts. In spite of this complete peripheral twitch occlusion, electrical transcranial cortical stimulation evoked a large $P$ di response. It was larger when the efforts were expulsive than inspiratory, but existed in both cases. As was the case in our study, this "extra" $P$ di response at times exceeded the peripheral twitch at relaxed FRC, and, with expulsive manoeuvres, was predominantly due to a rise in gastric pressure.

To explain their data, GANDEviA et al. [9] emphasized the fact that maximal activation of the diaphragm, as ascertained by the twitch occlusion, was not mandatorily associated with maximal activation of its synergistic in-series muscles (e.g. the inspiratory intercostals in the case of inspiratory manoeuvres, or the abdominal muscles in the case of expulsive manoeuvres). These muscles, therefore, could still be recruited by CxMS in spite of maximal diaphragmatic activation, and could contribute to $P$ di if transmission of the corresponding $P_{\text {ga }}$ to the thorax or of $P$ oes to the abdomen was incomplete. Such an incomplete pressure transmission through the diaphragm was demonstrated by direct stimulation of abdominal muscles: their contraction did not produce $P$ di when the diaphragm was relaxed, but a $P$ di swing appeared when it was contracted. If this hypothesis is correct CxMS could provide an estimate of the degree of global activation of synergistic respiratory muscles during voluntary respiratory manoeuvres.

However, it should be noted that the RC and AB displacement data collected in subjects Nos. 6 and 7 in the present study, although very fragmentary and preliminary, could cast doubt on the theory presented above and bring the diaphragm back into play. Indeed, $P$ di,c responses at $P$ di,max were not associated with an inward movement of the abdominal wall, as would have been expected with abdominal recruitment, but rather with an outward movement suggesting a diaphragmatic contraction. There was also some degree of RC inward movement.

Suggesting a mechanism by which the diaphragm, supposedly fully activated, could still respond to CxMS, is not simple. Because we did not use the most sensitive technique [35] to ascertain the reality of twitch occlusion in our subjects, one could argue that diaphragmatic activation at the highest values of $P$ di,vol was not actually maximal. In this case, a positive $P$ di,c would be expected, but its amplitude would be very small. Alternatively, BES could have combined excitatory and inhibitory effects [36], which would limit the corresponding $P$ di output. The persistence of $P$ di,c would then be explained by the lack of inhibitory effects with CxMS. This remains highly speculative. Confirmation of the role of the diaphragm in development of $P_{\mathrm{di}, \mathrm{c}}$ at $P_{\mathrm{di}}$,max on a larger number of subjects on the one hand, and other types of investigation, such as collision tests, on the other hand are obviously needed to clarify this point.

Acknowledgements: The authors are indebted to $\mathrm{O}$. Leray for her kind and valuable technical assistance, to P. Bouche, and to S. Launois for her precious help with both the study and the manuscript.

\section{References}

1. Merton PA. Voluntary strength and fatigue. J Physiol (Lond) 1954; 67: 563-564.

2. Bellanger AY, McComas AJ. Extent of motor unit activation during effort. J Appl Physiol: Respirat Environ Exercise Physiol 1981; 51: 1131-1135.

3. Rutherford OM, Jones DA, Newham DJ. Clinical and experimental application of the percutaneous twitch superimposition technique for the study of human muscle activation. J Neurol Neurosurg Psychiatr 1986; 49: 1288-1291.

4. Agostoni E, Rahn H. Abdominal and thoracic pressures at different lung volumes. J Appl Physiol 1960; 15: 10871092.

5. Laporta D, Grassino A. Assessment of transdiaphragmatic pressure in humans. J Appl Physiol 1985; 58: 1469-1476.

6. Hershenson MB, Kikuchi Y, Loring SH. Relative strengths of the chest wall muscles. J Appl Physiol 1988; 65: 852862.

7. Bellemare F, Bigland-Ritchie B. Assessment of human diaphragmatic strength and activation using phrenic nerve stimulation. Respir Physiol 1984; 58: 263-277.

8. Gandevia SC, McKenzie DK. Activation of the human diaphragm during maximal static efforts. I Physiol (Lond) 1985; 367: 45-46.

9. Gandevia SC, McKenzie DK, Plassman BL. Activation of human respiratory muscles during different voluntary manoeuvres. J Physiol (Lond) 1990; 428: 387-403.

10. Merton PA, Hill DK, Morton HB. Indirect and direct stimulation of fatigued human muscle. In: Porter R, Whelan J, ed. Human Muscle Fatigue: Physiological Mechanisms. (CIBA foundation symposium No. 82). London, Pitman Medical, 1981; pp. 120-126.

11. Ferguson GT. Use of twitch pressures to assess diaphragmatic function and central drive. J Appl Physiol 1994; 77: 1705-1715.

12. Bigland-Ritchie B, Jones DA, Hosking GP, Edwards RHT. Central and peripheral fatigue in sustained maximum voluntary contractions of human quadriceps muscle. Clin Sci Mol Med 1978; 54: 609-614. 
13. McKenzie DK, Bigland-Ritchie B, Gorman RB, Gandevia SC. Central and peripheral fatigue of human diaphragm and limb muscles assessed by twitch interpolation. $J$ Physiol (Lond) 1992; 454: 643-656.

14. Allen GM, McKenzie DK, Gandevia SC, Bass S. Reduced voluntary drive to breathe in asthmatic subjects. Respir Physiol 1993; 93: 29-40.

15. Allen GM, Hickie I, Gandevia SC, McKenzie DK. Impaired voluntary drive to breathe: a possible link between depression and unexplained ventilatory failure in asthmatic patients. Thorax 1994; 49: 881-884.

16. Similowski T, Yan S, Gauthier AP, Macklem PT, Bellemare F. Contractile properties of the human diaphragm during chronic hyperinflation. N Engl J Med 1991; 325: 917-923.

17. NHLBI workshop. Respiratory muscle fatigue. Am Rev Respir Dis 1990; 142: 474-480.

18. Similowski T, Fleury B, Launois S, Cathala HP, Bouche P, Derenne JP. Cervical magnetic stimulation: a new and painless method for bilateral phrenic nerve stimulation in conscious humans. J Appl Physiol 1989; 67: 1311-1318.

19. Bland J, Altman D. Statistical methods for assessing agreement between two methods of clinical measurement. Lancet 1986; i: 307-310.

20. Maskill D, Murphy K, Mier A, Owen M, Guz A. Motor cortical representation of the diaphragm in man. J Physiol (Lond) 1991; 443: 105-121.

21. Wragg S, Hamnegard C, Road J, et al. Potentiation of diaphragmatic twitch after voluntary contraction in normal subjects. Thorax 1994; 49: 1234-1237.

22. Similowski T, Straus C, Derenne J-P. Cortical magnetic stimulation allows diaphragm activation in man without facilitation. Eur Respir J 1993; 6: 194s (Abstract).

23. Wragg S, Aquilina R, Moran J, et al. Comparison of cervical magnetic stimulation and bilateral percutaneous electrical stimulation of the phrenic nerves in normal subjects. Eur Respir J 1994; 7: 1788-1792.

24. Cros D, Chiappa KH, Gominak S, et al. Cervical magnetic stimulation. Neurology 1990; 40: 1751-1756.

25. Similowski T, Launois S, Whitelaw WA, Derenne J-P.
Origin and characteristics of diaphragm contraction induced by cervical magnetic stimulation. Am Rev Respir Dis 1993; 147: A699 (Abstract).

26. Moxham J, Shneerson JM. Diaphragmatic pacing. Am Rev Respir Dis 1993; 148: 533-536.

27. Kelley WO. Phrenic nerve paralysis: special consideration of the accessory phrenic nerve. J Thorac Surg 1950; 19: 923-928.

28. Maertens De Noordhout A, Pepin JL, Gerard P, Delwaide PJ. Facilitation of responses to motor cortex stimulation: effects of isometric voluntary contraction. Ann Neurol 1992; 32: 365-370.

29. Close RI. Dynamic properties of mammalian skeletal muscles. Physiol Rev 1972; 52: 129-197.

30. Murphy K, Mier A, Adams L, Guz A. Putative cerebral cortical involvement in the ventilatory response to inhaled $\mathrm{CO}_{2}$ in conscious man. J Physiol (Lond) 1990; 420: $1-18$.

31. Gea J, Espadaler JM, Guiu R, Aran X, Seoane L, Broquetas JM. Diaphragmatic activity induced by cortical stimulation: surface versus esophageal electrodes. $J \mathrm{Appl}$ Physiol 1993; 74: 655-658.

32. Lissens MA. Motor evoked potentials of the human diaphragm elicited through magnetic transcranial brain stimulation. J Neurol Sci 1994; 124: 204-207.

33. Hess CW, Mills KR, Murray NMF. Responses in small hand muscles from magnetic stimulation of the human brain. J Physiol (Lond) 1987; 388: 397-419.

34. Cowan JMA, Day BL, Marsden CD, Rothwell JC. The effect of percutaneous motor cortex stimulation on $\mathrm{H}-$ reflexes in muscles of the arm and leg in man. J Physiol (Lond) 1986; 377: 333-348.

35. Hales JP, Gandevia SC. Assessment of maximal voluntary contraction with twitch interpolation: an instrument to measure twitch responses. J Neurosci Methods 1988; 25: $97-102$.

36. Duron B, Jung-Caillol M, Marlot D. Réflexe inhibiteur phrénico-phrénique. In: Duron B, ed. Respiratory Centers and Afferent Systems. Paris, editions de l'Institut National de la Santé et de la Recherche Médicale, 1976; pp. 193-197. 